\title{
Multi-center pragmatic studies evaluating the time indicator of cardiac perfusion reserve"
}

\author{
Lin Xiong ${ }^{1}$, Shouzhong Xiao ${ }^{2 \#}$, Qiang Zhou ${ }^{1}$, Xianrong Wu ${ }^{1}$, Zifu Xiao ${ }^{3}$, Xingming Guo ${ }^{2}$, Delin $\mathrm{Lu}^{2}$, \\ Wanrong $\mathrm{Zhao}^{4}$, Xiaojun $\mathrm{Wu}^{4}$, Xiaobo Yan ${ }^{5}$, Yanxia Zhao ${ }^{5}$, Jianming Liu
}

\author{
${ }^{1}$ Chongqing Qingmuguan Central Hospital, Chongqing, China \\ ${ }^{2}$ Department of Biomedical Engineering, Chongqing University, Chongqing, China \\ ${ }^{3}$ Chongqing Bo-Jing Medical Informatics Institute, Chongqing, China \\ ${ }^{4}$ The First Affiliated Hospital of Chongqing Medical University, Chongqing, China \\ ${ }^{5}$ Institute of Physical Education, Chongqing University, Chongqing, China \\ Email: ${ }^{\text {xiaoshouzhong@hotmail.com }}$
}

Received 18 October 2012; revised 19 November 2012; accepted 7 December 2012

\begin{abstract}
Background: Since the greater part of coronary blood flow takes place during the diastolic phase of each cardiac cycle, a time indicator of myocardial perfusion reserve, the ratio of diastolic to systolic duration $(\mathrm{D} / \mathrm{S}$ ratio), was presented. The objective of this study was to evaluate the accuracy and precision, the biological implication, and the applications of $\mathrm{D} / \mathrm{S}$ ratio. Methods: Multi-center pragmatic studies evaluating the time indicator of cardiac perfusion reserve were performed. Related experiments, clinical trials, and surveys were conducted at 5 centers. Results: The results showed that the measurement of $\mathrm{D} / \mathrm{S}$ ratio is both accurate and precise; the mean values of $D / S$ of all of the 3 species studied (human, rabbit, and rat) were greater than 1. These application studies on $\mathrm{D} / \mathrm{S}$ ratio showed that a close negative correlation existed between $\mathrm{D} / \mathrm{S}$ ratio and New York Heart Association Functional Classification (NYHA FC) $(r=-0.659, p<0.01)$; normal persons were mostly distributed at NYHA FC I and at high value of $\mathrm{D} / \mathrm{S}$ ratio; the patients with cardiovascular disease were mostly at low value of $D / S$ ratio; the difference of $D / S$ between pregnant women with preeclampsia and either normal pregnant women or nonpregnant women were significant $(p<0.05)$; athletes had higher $\mathrm{D} / \mathrm{S}$ ratio than non-athletes $(2.04 \pm 0.33$ vs $1.82 \pm 0.27, p<0.01)$. Conclusions: $\mathrm{D} / \mathrm{S}$ ratio has important biological implication, which is a safe, easy, reliable, and effective indicator, can be used to evaluate fitness levels, served as a pathophysiological marker for screening of cardiovascular disease (CVD), for predicting risk of cardiac events, and for evaluating the severity and prognosis of CVD.
\end{abstract}

*The authors declare that they have no competing interests.

${ }^{\#}$ Corresponding author.
Keywords: Ratio of Diastolic to Systolic Duration (D/S); Accuracy; Precision; Cardiac Reserve; Cardiac Safety; Fitness

\section{INTRODUCTION}

There are at least two major determinants for coronary perfusion: coronary dimension and coronary perfusion time. As described by Abe et al., the greater part of coronary blood flow takes place during the diastolic phase of each cardiac cycle [1]. Based on the above, we presented an indicator $\mathrm{D} / \mathrm{S}$ ratio (the ratio of diastolic to systolic duration) to evaluate whether the myocardial perfusion time is sufficient or not [2], which is an indicator of cardiac reserve, because it relates to how much nutrition and oxygen will be available during systole, and relates to ventricular filling and cardiac output.

Heart sound signal is the direct manifestation of cardiac mechanical activity, which can safely, easily, and accurately provide the information on cardiac cycle and the durations of its components. So heart sound signal was analyzed to obtain $\mathrm{D} / \mathrm{S}$ ratio in this study. The cutoff value of $\mathrm{D} / \mathrm{S}$ ratio for adults was determined by using receiver operating characteristic curve analysis, which was $1.5[3]$.

This paper describes the establishment of the time indicator of cardiac perfusion reserve, $\mathrm{D} / \mathrm{S}$ ratio, and its applications in multiple fields.

\section{MENTHODS AND SUBJECTS}

\subsection{Measurement of $D / S$ Ratio}

Cardiac Contractility Monitor (CCM, Bojing Medical Informatics Institute, Chongqing) was used as the signal collection and processing device, which is comprised of a phonocardiographic sensor (Beijing Space Medicine 
Research Institute), a preprocessing circuit, and a personal computer. The preprocessing circuit includes a band pass filter with a passband of $30 \mathrm{~Hz}$ to $800 \mathrm{~Hz}$ and a variable gain amplifier, which is connected with a personal computer. In order to popularize the applications of our heart sound database resources, Visual Basic and Visual Fox Pro were used to create database and write program.

Signal collection: Heart sounds (sampling at 11.025 $\mathrm{kHz}$ ) were recorded at the apex for all subjects. The preprocessed signals were digitized with 8-bit resolution and stored on a hard disk of the PC for later analysis. The data were saved as WAV files. The WAV files were saved in a folder in the computer.

Waveform measurement and statistical analysis: the amplitude and duration of each component for each heart sound sample were measured by using heart sound analysis software developed by our team. The data from these measurements were used to generate relevant indicators. Quantitative data are presented as mean $\pm \mathrm{SD}$. Statistical comparisons between groups were performed with a $t$-test. A value of $p<0.05$ was considered to be statistically significant. Statistical analysis was performed with SPSS software (SAS Institute, Inc.).

\subsection{Validation of the Measurements of $D / S$ Ratio}

The following measurements were performed at Qingmuguan Hospital, Chongqing.

The time measurement of various components of a cardiac cycle was based on the technique criteria of computer clock and PC's built-in recording software. The sampling rate was set at $11,025 \mathrm{~Hz}$.

The accuracy and precision of measurements of $D / S$ ratio were evaluated by performing the following studies.
The results are summarized in Table 1.

1) Based on the computer clock, a phonocardiogram (PCG) wave model was constructed by Dr. Hong Tang (Dalian University of Technology), which was served as the reference for evaluating the accuracy of the measured values of $\mathrm{D} / \mathrm{S}$ ratio measured by Cardiac Contractility Monitor (CCM). The parameters of the PCG wave model are: diastole $=0.48 \mathrm{~s}$, systole $=0.32 \mathrm{~s}$, and $\mathrm{D} / \mathrm{S}$ ratio $=$ 1.50. When measuring the PCG wave model using CCM 10 times, mean $\mathrm{D} / \mathrm{S}$ ratio was $1.4972 \pm 0.0057$, and the mean difference between the measured values by CCM and the parameter value of D/S ratio of PCG wave model was $0.0028 \pm 0.0056$. The results show that the accuracy of using $\mathrm{CCM}$ to measure $\mathrm{D} / \mathrm{S}$ ratio is high.

2) A comparison was undertaken to evaluate the accordance of the measured values of $\mathrm{D} / \mathrm{S}$ ratio obtained during a short period of time and from the PCG samples simultaneously recorded at each paired auscultation areas, mitral (M) respectively versus tricuspid (T), aortic (A), pulmonary $(\mathrm{P})$, and Erb (E) areas. The measured values of $\mathrm{D} / \mathrm{S}$ ratio for various paired auscultation areas, and the corresponding differences $(\Delta \mathrm{D} / \mathrm{S})$ are shown in Table 1. The mean difference value of $\mathrm{D} / \mathrm{S}$ ratios for all paired areas was $0.0216 \pm 0.0197$, suggesting the accordance of $\mathrm{D} / \mathrm{S}$ ratios obtained from the four paired auscultation areas is good.

3) A comparison was conducted to evaluate the repeatability of the measured values of $\mathrm{D} / \mathrm{S}$ ratio obtained by one researcher, who performed repeated measurements 10 times for the same cardiac cycle on the same PCG sample. The mean value, SD, and coefficient of variation $(\mathrm{CV})$ of $\mathrm{D} / \mathrm{S}$ ratios were $1.87 \pm 0.0066$ and $0.35 \%$, indicating that the repeatability was good.

Table 1. The accuracy and precision of the measurement of $\mathrm{D} / \mathrm{S}$ ratio.

\begin{tabular}{|c|c|c|c|c|c|c|c|}
\hline \multicolumn{2}{|c|}{ Measurements } & $\mathrm{D} / \mathrm{S}$ & $\mathrm{D} / \mathrm{S}$ at $\mathrm{M}$ & $\Delta \mathrm{D} / \mathrm{S}$ & $\mathrm{CV}, \%$ & $\mathrm{~F}$ & p-value \\
\hline \multicolumn{2}{|c|}{ PCG Model $(\mathrm{D} / \mathrm{S}=1.50)$ measured by $\mathrm{CCM}$} & $1.4972 \pm 0.0057$ & & $0.0028 \pm 0.0056$ & 0.38 & & \\
\hline \multirow{3}{*}{$\begin{array}{l}\text { Areas paired } \\
\text { with } \mathrm{M}\end{array}$} & Tricuspid area & 1.7006 & 1.7015 & 0.0009 & & & \\
\hline & Aortic area & 1.877 & 1.831 & 0.046 & & & \\
\hline & Erb area & 1.7523 & 1.7244 & 0.0279 & & & \\
\hline $\mathrm{M}$ area alone & & $1.87 \pm 0.0066$ & & & 0.35 & & \\
\hline \multirow{2}{*}{$\begin{array}{c}\text { Same cardiac } \\
\text { Cycle measured by }\end{array}$} & Researcher A & $1.4972 \pm 0.0057$ & & \multirow{2}{*}{0.0038 (A versus B) } & 0.38 & \multirow{2}{*}{1.1241} & \multirow{2}{*}{0.4323} \\
\hline & Researcher B & $1.4934 \pm 0.0059$ & & & 0.4 & & \\
\hline \multirow{2}{*}{$\mathrm{CC} 1$ vs CC2 } & $\mathrm{CC} 1$ & $2.09 \pm 0.0092$ & & & 0.44 & \multirow{2}{*}{1.9962} & \multirow{2}{*}{0.1589} \\
\hline & $\mathrm{CC} 2$ & $1.87 \pm 0.0066$ & & & 0.35 & & \\
\hline
\end{tabular}

PCG: phonocardiogram; D/S: the ratio of diastolic to systolic duration; CCM: Cardiac Contractility Monitor; M: mitral area ; CC: cardiac cycle; CV: coefficient of variation. 
4) A comparison was performed to evaluate the consistency of measured values of $\mathrm{D} / \mathrm{S}$ ratio obtained from the same cardiac cycle on the same PCG but measured 10 time, respectively by researcher A and researcher B (came from different laboratories). The mean value and $\mathrm{SD}$ of $\mathrm{D} / \mathrm{S}$ ratios were $1.4972 \pm 0.0057$ and $1.4934 \pm$ 0.0059 (A versus B), respectively, the difference value of $\mathrm{D} / \mathrm{S}$ was 0.0038 (A versus $\mathrm{B}$ ), and the accordance of $\mathrm{D} / \mathrm{S}$ ratios measured by $\mathrm{A}$ and $\mathrm{B}$ was high $(\mathrm{F}=1.1241, \mathrm{p}=$ 0.4323).

5) A comparison was conducted to evaluate the replicability of measured values of $\mathrm{D} / \mathrm{S}$ ratio obtained by one researcher, who performed repeated measurements 10 times for two cardiac cycles $(\mathrm{C} 1$ and $\mathrm{C} 2)$ of the same PCG sample. The SD and coefficient of variation (CV) of $\mathrm{D} / \mathrm{S}$ ratio were $\pm 0.0092,0.44 \%$ and $\pm 0.0066,0.35 \%$ (C1 versus $\mathrm{C} 2$ ), respectively, indicating that the replicability was satisfactory $(\mathrm{F}=1.9962, \mathrm{p}=0.1589)$.

\subsection{Three Species}

To explore the biological implication of $\mathrm{D} / \mathrm{S}$ ratio, the following measurements were performed in 3 species.

1) Humans: One hundred and twenty-three adults without cardiovascular disease were included in this survey. This study was approved by the Institutional Review Board of Chongqing University, and informed consent was obtained from all subjects.

2) Animals: Eight New Zealand White rabbits (1.5 to 2 $\mathrm{kg}, 4$ females) and 10 male Wistar rats (2 months old) (Experimental Animal Centre of the Third Military Medical University) were included in this study. Experimental protocols for this study were approved by the Ethics Committee of Institute of Physical Education, Chongqing University. All animal care and use programs were carried out according to the Regulations for the Administration of Affairs Concerning Experimental Animals of Chongqing.

\subsection{Three Populations}

$\mathrm{D} / \mathrm{S}$ ratio indicator has been used in multiple fields. The following studies were performed in 3 populations at 3 institutions.

\subsubsection{Patients with Cardiovascular Diseases (CVD)}

To evaluate the cardiac function of patients with cardiovascular diseases, a clinical trial was carried out at Chongqing Medical University, Chongqing City.

In order to explore the indicators and method for a comprehensive evaluation of cardiac function of patients with cardiovascular diseases (CVD), a clinical trial was carried out. A total of 182 consecutive subjects were enrolled in this study, 136 of whom with CVD, aged 50 to 85 years $(62.20 \pm 12.39), 78$ men, including 59 with coronary heart disease, 56 hypertension, 13 valvular heart disease, 6 dilated cardiomyopathy, 2 hyperthyroid heart disease, who were in New York Heart Association functional class (NYHA-FC) I ( $\mathrm{n}=35)$, II(52), III(36), and IV(13), respectively; 46 volunteers without CVD served as controls, aged 50 to 75 years $(61.28 \pm 11.39)$, 24 men.

The ratio of diastolic to systolic duration (D/S ratio) was detected. The diagnostic value of $\mathrm{D} / \mathrm{S}$ ratio, 6-minute walk distance (6MWD), and Left ventricular ejection fraction (LVEF) for CVD was assessed, respecttively. In order to analyze the benefit of $\mathrm{D} / \mathrm{S}$ ratio for evaluating the severity of CVD, the distribution of $\mathrm{D} / \mathrm{S}$ ratio of subjects over the NYHA-FC was investigated.

\subsubsection{Pregnant Women}

Pregnancy increases cardiac burden. The total cardiac burden of the pregnant woman includes not only pregnancy but a great variety of other burdens. Under some conditions, a total cardiac burden during pregnancy may exceed the maximal limit of the cardiac reserve of a pregnant woman, and a problem of cardiac safety may arise. The aim of this survey was to obtain statistical values of several indicators, especially the $\mathrm{D} / \mathrm{S}$ ratio, for evaluation of pregnant women at risk of cardiac events.

One hundred and eighty-eight women were consecutively enrolled in the survey, including 71 non-pregnant women, 94 normal pregnant women, and 23 women with pre-eclampsia. The survey was carried out at the Maternal and Child Health Hospital of Liangping and Chongqing Medical University, Chongqing City.

\subsubsection{Athletes}

Cardiac reserve is one of the most important physiological bases of athletes' fitness and exercise performance. There is a 20 -fold difference between the most impaired cardiac function and that of the fittest person [4]. The ratio of the amplitude of the first heart sound to the amplitude of the second heart sound (S1/S2) can be used to evaluate left ventricular systolic function, especially the cardiac contractility reserve [3]. A study on the association between $\mathrm{D} / \mathrm{S}$ ratio and fitness was carried out. Eighty-one athletes (59 males and 22 females, aged 17 to 27 years) and 41 non-athletes ( 29 males and 12 females, aged 21 to 29 years) were voluntarily enrolled in this study. Tests were performed at Cardiac Reserve Laboratory, Chongqing University.

\section{RESULTS}

\subsection{Results of Measurement of $D / S$ Ratio for 3 Species}

Data of measurement of $\mathrm{D} / \mathrm{S}$ ratio for 3 species are shown in Table 2. 


\subsection{Evaluation of Cardiac Function}

A table of the distribution of $\mathrm{D} / \mathrm{S}$ ratio over the cardiac function classification (New York Heart Association Functional Classification, NYHA FC) is constructed (Table 3). Normal persons were mostly distributed at NYHA FC I and at high value of D/S ratio; the patients with cardiovascular disease were mostly at low value of $\mathrm{D} / \mathrm{S}$ ratio.

Comparison of 6MWD, D/S and LVEF between CVD Group and Controls is shown in Table 4.

The correlation of $\mathrm{D} / \mathrm{S}, 6 \mathrm{MWD}$, and $\mathrm{LVEF}$ with NYHA FC is shown in Table 5.

\subsection{A Survey for Evaluation of Pregnant Women at Risk for Cardiac Events}

As shown in Table 6, the differences of D/S and HR between pregnant women with pre-eclampsia and either normal pregnant women or non-pregnant women were significant $(p<0.05)$. With the increase in the number of weeks of pregnancy and progress of pre-eclampsia, the

Table 2. Data of measurement of $\mathrm{D} / \mathrm{S}$ ratio for 3 species.

\begin{tabular}{cc}
\hline Species (n) & D/S ratio (mean \pm SD) \\
\hline Humans (123) & $1.64 \pm 0.26$ \\
Rabbits (8) & $1.28 \pm 0.06$ \\
Rats (10) & $1.57 \pm 0.16$ \\
\hline
\end{tabular}

$\mathrm{D} / \mathrm{S}$ ratio: the ratio of diastolic to systolic duration.

Table 3. The distribution of $\mathrm{D} / \mathrm{S}$ ratio of 182 subjects over the cardiac function classification.

\begin{tabular}{cccccc}
\hline \multirow{2}{*}{ Groups (n) } & NYHA & \multicolumn{5}{c}{ D/S } \\
\cline { 3 - 6 } & FC(n) & $\geq 1.5$ & $1.49-1.30$ & $1.29-1.00$ & $<1.00$ \\
\hline Controls (46) & I (46) & 44 & 2 & 0 & 0 \\
& I (35) & 18 & 12 & 5 & 0 \\
Patients & II (52) & 13 & 31 & 6 & 2 \\
with CVD & III(36) & 4 & 9 & 19 & 4 \\
(136) & IV(13) & 0 & 0 & 3 & 10 \\
\hline
\end{tabular}

NYHA FC: New York Heart Association Functional Classification; D/S: the ratio of diastolic to systolic duration; CVD: cardiovascular diseases.

Table 4. Comparison of 6MWD, D/S and LVEF between CVD Group and Controls.

\begin{tabular}{ccc}
\hline Indicators & CVD Group $(\mathrm{n}=136)$ & Controls $(\mathrm{n}=46)$ \\
\hline D/S & $1.35 \pm 0.33^{*}$ & $1.72 \pm 0.22$ \\
6MWD (m) & $392.9 \pm 93.70^{*}$ & $487.1 \pm 54.20$ \\
LVEF (\%) & $61 \pm 12.31^{*}$ & $63.2 \pm 1.4$ \\
\hline
\end{tabular}

CVD: cardiovascular diseases; D/S: the ratio of diastolic to systolic duration; 6MWD: 6-minute walk distance; LVEF: Left ventricular ejection fraction; ${ }^{*} \mathrm{p}<0.05$ versus controls. number of subjects with $\mathrm{D} / \mathrm{S}$ ratio $\geq 1.5$ was decreased and the number of subjects with $\mathrm{D} / \mathrm{S}$ ratio $<1.2$ was increased. Notably, a pregnant woman with $\mathrm{D} / \mathrm{S}$ of 0.59 died of left heart failure $2 \mathrm{~h}$ after the first examination, and another pregnant woman with $\mathrm{D} / \mathrm{S}$ of 0.87 was sent to intensive care unit for heart failure.

\subsection{Evaluation of Fitness}

$\mathrm{D} / \mathrm{S}, \mathrm{S} 1 / \mathrm{S} 2$, and $\mathrm{HR}$ of 81 athletes and 41 non-athletes are shown in Table 7.

\section{DISCUSSION}

\subsection{The Accuracy and Precision of Measurements of $\mathrm{D} / \mathrm{S}$ Ratio}

The data summarized in Table $\mathbf{1}$ indicate that the accuracy and precision of measurements of $\mathrm{D} / \mathrm{S}$ ratio is high, including the repeatability, reproducibility, and replicability. Especially the accordance of $\mathrm{D} / \mathrm{S}$ ratios obtained

Table 5. The correlation of $\mathrm{D} / \mathrm{S}, 6 \mathrm{MWD}$, and LVEF with NYHA FC.

\begin{tabular}{ccc}
\hline & $\mathrm{r}$ & $\mathrm{p}$ \\
\hline $\mathrm{D} / \mathrm{S}$ & -0.659 & $<0.01$ \\
6MWD & -0.561 & $<0.01$ \\
$\mathrm{LVEF}$ & 0.203 & $<0.05$ \\
\hline
\end{tabular}

D/S: the ratio of diastolic to systolic duration; 6MWD: 6-minute walk distance; LVEF: Left ventricular ejection fraction. The data of Table 5 came from 182 study subjects admitted into the cardiological wards of the Chongqing Medical University mentioned in the manuscript.

Table 6. Statistical data of D/S and HR of 188 women.

\begin{tabular}{cccc}
\hline Subjects & $\mathrm{n}$ & $\mathrm{D} / \mathrm{S}$ & HR (beats/min) \\
\hline Non-pregnant women & 71 & $1.57 \pm 0.32$ & $76 \pm 10$ \\
Normal pregnant women & 94 & $1.42 \pm 0.25$ & $85 \pm 13$ \\
Pre-eclampsia & 23 & $1.12 \pm 0.31^{*}$ & $96 \pm 19^{*}$ \\
\hline
\end{tabular}

$\mathrm{D} / \mathrm{S}$ : ratio of diastolic to systolic duration; HR: heart rate; ${ }^{*} \mathrm{p}<0.05$ vs either non-pregnant women or normal pregnant women.

Table 7. D/S, S1/S2, and HR of 81 athletes and 41 non-athletes.

\begin{tabular}{cccc}
\hline & $\begin{array}{c}\text { Athletes } \\
(\mathrm{n}=81)\end{array}$ & $\begin{array}{c}\text { Nonathletes } \\
(41)\end{array}$ & p-value \\
\hline $\mathrm{D} / \mathrm{S}$ at rest & $2.04 \pm 0.33$ & $1.82 \pm 0.27$ & $<0.01$ \\
$\mathrm{~S} 1 / \mathrm{S} 2$ at rest & $1.51 \pm 0.76$ & $1.01 \pm 0.38$ & $<0.001$ \\
$\mathrm{~S} 1 / \mathrm{S} 2$ after exercise & $7.34 \pm 4.04$ & $5.27 \pm 2.40$ & $<0.01$ \\
HR at rest & $68 \pm 9$ & $71 \pm 7.98$ & $<0.0006$ \\
HR after exercise & $159 \pm 11$ & $166 \pm 11$ & 0.001 \\
\hline
\end{tabular}

$\mathrm{D} / \mathrm{S}$ : the ratio of diastolic to systolic duration; $\mathrm{S} 1 / \mathrm{S} 2$ : the ratio of the amplitude of the first heart sound to the amplitude of the second heart sound; HR: heart rate. 
from the four paired auscultation areas is good, suggesting that any of these auscultation areas can be adopted for measurements of $\mathrm{D} / \mathrm{S}$ ratio if any other auscultation area is not appropriate.

\subsection{The Biological Implication of $D / S$ Ratio}

Table 2 shows that the mean values of $\mathrm{D} / \mathrm{S}$ of all of the 3 species were greater than 1, i.e., the diastolic duration is longer than the systolic duration. Since the greater part of coronary blood flow takes place during the diastolic phase of each cardiac cycle [1], higher D/S ratio is beneficial to myocardial perfusion, thus helping maintain the cardiac health status. Maybe $\mathrm{D} / \mathrm{S}>1$ is an evolutionary characteristic of mammal, being beneficial to their physical activity and survival. The swimming time to exhaustion for rabbits was several to a dozen minutes, and for rats, several hours. This condition may be related to the fact that rabbits have a $\mathrm{D} / \mathrm{S}$ ratio of only $1.28 \pm$ 0.06 , whereas rats have a $\mathrm{D} / \mathrm{S}$ ratio of $1.57 \pm 0.16$.

\subsection{Applications of $D / S$ Ratio Indicator}

The data shown in Tables 3 and $\mathbf{5}$ indicate that the accordance between $\mathrm{D} / \mathrm{S}$ ratio and NYHA FC is good. D/S ratio may be a practical indicator for more comprehendsive evaluation of cardiac function and the severity of CV disease.

Whether the time during diastole is sufficient or not relates to how much nutrition and oxygen will be available for the next cardiac contraction, so D/S ratio is considered as an indicator of cardiac reserve. $\mathrm{D} / \mathrm{S}$ ratio $<1$ should be served as a warning value, indicating possible cardiac events, including sudden death [5]. During a one-year follow-up, there were 3 deaths among 32 subjects with $\mathrm{D} / \mathrm{S}<1.5$, whereas none of the 140 subjects with $\mathrm{D} / \mathrm{S} \geq 1.5$ died [2].

The results of the survey for evaluation of pregnant women at risk for cardiac events show that the indicator $\mathrm{D} / \mathrm{S}$ ratio, which is based on the non-invasive method above-mentioned, can be used as an indicator for evaluating the cardiac safety of pregnant women; a value of $\mathrm{D} / \mathrm{S}$ ratio $<1$ can be considered as a warning value for risk of cardiac events. In general, $\mathrm{D} / \mathrm{S}$ ratio of nonpregnant women is rarely less than 1.5 , none with $\mathrm{D} / \mathrm{S}$ ratio $<1[5]$.

The above situations would support that $\mathrm{D} / \mathrm{S}$ ratio is beneficial for evaluating the severity and prognosis of $\mathrm{CV}$ disease, can be served as a pathophisiological marker for screening $\mathrm{CV}$ diseases and for predicting risk of cardiac events.

Our team presented the indicator D/S ratio, systematically studied it, and the statistical data were published in 2002 [2]. Before 2002, there was no report of D/S ratio in PubMed. Although Iuchi, A. et al. also used the term
$\mathrm{D} / \mathrm{S}$ ratio in 1991 , their $\mathrm{D} / \mathrm{S}$ ratio referred to the ratio of diastolic to systolic peak velocity in pulmonary venous, not to the ratio of diastolic to systolic duration of the heart. The $\mathrm{D} / \mathrm{S}$ ratio reflects cardiac chronotropic state and the S1/S2 ratio reflects cardiac inotropic state. Inotropism and chronotropism are two aspects of cardiac properties.

Table 7 shows that, during exercise, the S1/S2 ratio of the athletes can increase to a higher level $(7.34 \pm 4.04)$ than the non-athletes $(5.27 \pm 2.40)$, which means that athletes have a higher cardiac contractility reserve than non-athletes.

The data shown in Table 7 support that there is a close association between D-S ratio and fitness. We previously performed a statistical analysis using a database created from a heart survey for 81 footballers [5]. The D-S ratios for them were $1.82 \pm 0.36$. Notably, of them, $23(28 \%)$ had a D-S ratio equal to or greater than 2.0, which was significantly greater than that of a general populationbased sample $(1.68 \pm 0.27, n=172)$ [2]. It means that exercise can enhance $\mathrm{D}-\mathrm{S}$ ratio.

Our team also studied the application of $\mathrm{D} / \mathrm{S}$ ratio indicator in other fields, such as in preterm infants [6], in neonates [7], in healthy children aged $1-18$ years $[5,8,9]$, in children with atrial septal defect [10], in coal miners [11], in pregnant women [12-14], and in middle-aged and elderly person [15]. The corresponding data of $\mathrm{D} / \mathrm{S}$ ratio were obtained, which have been used to evaluate cardiac functional status in healthy persons or patients.

In 1995, one study involved S/D (not D/S) [16], the ratio of systolic (S) duration to diastolic (D) duration; in 2006 and since then, more researchers have increasingly been interested in studies involving S/D [17-22]. The calculation of S/D ratio was based on the Doppler-derived mitral or tricuspid regurgitation (MR/TR) signals. Doppler flow signals of atrioventricular regurgitation were acquired from the apical 4-chamber view by continuous-wave Doppler.

The S/D ratio was found to be abnormally increased in children with dilated and restrictive cardiomyopathy $[17,18]$. Friedberg and Silverman [19] investigated the $\mathrm{S} / \mathrm{D}$ duration ratio in children with hypoplastic left heart syndrome (HLHS) as a novel promising index of global $\mathrm{RV}$ function. At resting heart rates, systole (S) constitutes about $40 \%$ of the cardiac cycle in healthy children [19]. S/D duration was also proposed as an indicator of PAH severity [20,21]. Alkon et al. found that an increased S/D duration ratio $>1.4$ inversely correlates with survival in children with PAH [20]. They concluded that, in children with PAH, an increased S/D ratio is temporally associated with worse RV function, hemodynamics, exercise capability, and survival. $\mathrm{S} / \mathrm{D}$ duration ratio is beneficial for more detailed assessments of ventricular performance. 
Although S/D also reflects the relationship between diastolic and systolic durations, D/S highlights the importance of diastolic duration, and the normal value of $\mathrm{D} / \mathrm{S}$ is almost always greater than 1 , which is beneficial for quickly understanding the meaning of the measured value of $\mathrm{D} / \mathrm{S}$ ratio. A limitation of $\mathrm{S} / \mathrm{D}$ duration ratio is that the measurement of this ratio to assess ventricular function requires the presence of defined onset and end of MR/TR on spectral Doppler tracings [22]. In the study by Alkon et al. [20], 7 of 54 patients (13\%) were excluded because of inadequate TR Doppler tracing to measure the duration of systole and diastole. They reviewed 503 serial echocardiograms from these patients, of which the S/D ratio could be calculated in $382(76 \%)$. On $24 \%$ of echocardiograms there was inadequate TR to calculate the S/D ratio and this is an important limitation of the technique.

The essence of the two indicators, $\mathrm{D} / \mathrm{S}$ ratio and $\mathrm{S} / \mathrm{D}$ ratio, is the same that both of them can reflect a change in cardiac perfusion time. Since $\mathrm{D} / \mathrm{S}$ ratio and $\mathrm{S} / \mathrm{D}$ ratio are mutually reciprocal, the data of $\mathrm{D} / \mathrm{S}$ ratio and $\mathrm{S} / \mathrm{D}$ ratio resulted from the studies with the two methods can be easily shared by researchers, clinicians, and coaches, no matter which technic was used.

\section{CONCLUSION}

In brief, $\mathrm{D} / \mathrm{S}$ ratio has important biological implication, which is a safe, easy, reliable, and effective indicator for evaluating the severity and prognosis of $\mathrm{CV}$ disease, can be served as a pathophisiological marker for screening $\mathrm{CV}$.

\section{AUTHORS' CONTRIBUTIONS}

S.X. conceived of the study, participated in its design and coordination, and drafted the manuscript, L.X., Q.Z., X.W., and X.G. participated in the design, Z.X. participated in the evaluation of the accuracy and precision of measurements of $\mathrm{D} / \mathrm{S}$ ratio and clinical trial, D.L., W.Z., Q.Z., and X.W. participated in clinical trial, X.Y., Y.Z., and J.L. participated in the survey for evaluation of fitness of athletes and non-athletes and performed the animal experiment. All authors read and approved the final manuscript.

\section{ACKNOWLEDGEMENTS}

This study is partly supported by the National Natural Science Foundation of China (NO.30770551) and Chongqing Science \& Technology Commission (CSTC, 2008AC5103).

\section{REFERENCES}

[1] Abe, M., Tomiyama, H., Yoshida, H. and Doba, N. (2000)
Diastolic fractional flow reserve to assess the functional severity of moderate coronary artery stenoses: Comparison with fractional flow reserve and coronary flow velocity reserve. Circulation, 102, 2365-2370. doi:10.1161/01.CIR.102.19.2365

[2] Xiao, S., Guo, X., Sun, X. and Xiao, Z. (2002) A relative value method for measuring and evaluating cardiac reserve. BioMedical Engineering OnLine, 1, 6. doi:10.1186/1475-925X-1-6

[3] Xiao, S., Guo, X., Wang, F., Xiao, Z., Liu, G., Zhan, Z. and Sun, X. (2003) Evaluating two new indicators of cardiac reserve. IEEE Engineering in Medicine and Biology Magazine, 22, 147-152.

doi:10.1109/MEMB.2003.1237516

[4] Cooke, G.A., Marshall, P., Al-Timman, J.K., Wright, D.J., Riley, R., Hainsworth, R. and Tan, L.B. (1998) Physiological cardiac reserve: Development of a non-invasive method and first estimates in man. Heart, 79, 289-294.

[5] Xie, M., Xiao, S., Liu, T., Yi, Q., You, F., Guo, X., Shao, Y., Huo, J., Du, D., Xu, D., Wu, W., Xiao, Z., Yang, Y. and Guo, W. (2012) Multi-center, multi-topic heart sound databases and their applications. Journal of Medical Systems, 36, 33-40. doi:10.1007/s10916-010-9443-x

[6] Yang, X. and Zeng, W. (2011) Determination of cardiac reserve in preterm infants. Turkish Journal of Pediatrics, 53, 308-313.

[7] Yang, X. and Zeng, W. (2010) A relative value method for measuring and evaluating neonatal cardiac reserve. Indian Journal of Pediatrics, 77, 661-664. doi:10.1007/s12098-010-0058-5

[8] Huang, G., Xin, H. Shi, L., Huang, G., Xiao, Z., Xie, M., Yang, Y. and Xiao, S. (2010) A preliminary survey on two new indicators of infantile cardiac reserve. Laboratory Medicine and Clinic, 7, 1304-1308.

[9] Yang, Z., Li, C. and Xiao, S. (2003) A survey of three new non-invasive indicators of cardiac reserve of $6-12$ years old children. Chinese Journal of Medical Research, 3, 388-390.

[10] Huo, J., Yi, Q., Xiao, S., Xie, M. and Xiao, Z. (2011) Analysis of cardiac reserve in children with ASD before and after intervention. Journal of Chongqing Medical University, 36, 203-205.

[11] Li, C., Li, M., Jiang, M., Zhou, Y., Yang, L., Wei, X. and Xiao, S. (2003) A study of the heart reserve index in the coal miners. Journal of Occupational Health and Damage, 18, 85-87.

[12] Shao, Y., Zhang, Y. and Liu, O. (2012) Using phonocardiography to investigate maternal cardiac reserve function in gestational hypertension and pre-eclampsia. Journal of Obstetrics and Gynaecology Research, 39, 53-60. doi:10.1111/j.1447-0756.2012.01897.x

[13] Zhang, Y., Shao, Y., Xiao, S. and Guo, X. (2010) A clinical study of cardiac reserve mobilizing condition for pregnant women. Journal of Biomedical Engineering, 27, 1224-1228.

[14] Shao, Y., Zhang, Y., Qi, H., Xiao, S. and Guo, X. (2009) Clinical analysis of cardiac reserve function and outcome of preeclampsia. Chinese Journal of Obstetrics and Gy- 
necology, 44, 736-739.

[15] Xiao, S., Fang, X., Zhan, Z., Sun, X. and Xiao, Z. (2002) A clinical study in middle-aged and elderly persons on heart itself blood supply time during diastole with a noninvasive method. Chinese Journal of Medicine, 2, 961962.

[16] Fraser, G.E., Luke, R., Thompson, S., Smith, H., Carter, S. and Sharpe, N. (1995) Comparison of echocardiographic variables between type I diabetics and normal controls. American Journal of Cardiology, 15, 141-145. doi:10.1016/S0002-9149(00)80063-6

[17] Friedberg, M.K. and Silverman, N.H. (2006) Cardiac ventricular diastolic and systolic duration in children with heart failure secondary to idiopathic dilated cardiomyopathy. American Journal of Cardiology, 97, 101-105. doi:10.1016/j.amjcard.2005.07.127

[18] Friedberg, M.K. and Silverman, N.H. (2006) The systolic to diastolic duration ratio in children with heart failure secondary to restrictive cardiomyopathy. Journal of the American Society of Echocardiography, 19, 1326-1331. doi:10.1016/j.echo.2006.05.024
[19] Friedberg, M.K. and Silverman, N.H. (2007) The systolic to diastolic duration ratio in children with hypoplastic left heart syndrome: A novel Doppler index of right ventricular function. Journal of the American Society of Echocardiography, 20, 749-755. doi:10.1016/j.echo.2006.11.014

[20] Alkon, J., Humpl, T., Manlhiot, C., McCrindle, B.W., Reyes, J.T. and Friedberg, M.K. (2010) Usefulness of the right ventricular systolic to diastolic duration ratio to predict functional capacity and survival in children with pulmonary arterial hypertension. American Journal of Cardiology, 106, 430-436. doi:10.1016/j.amjcard.2010.03.048

[21] Sehgal, A., Athikarisamy, S.E. and Adamopoulos, M. (2012) Global myocardial function is compromised in infants with pulmonary hypertension. Acta Paediatrica, 101, 410-413. doi:10.1111/j.1651-2227.2011.02572.x

[22] Koestenberger, M., Friedberg, M.K., Ravekes, W. and Nestaas, E. (2012) Non-invasive imaging for congenital heart disease: Recent innovations in transthoracic echocardiography. Journal of Clinical \& Experimental Cardiology, $\mathbf{S 8}, 2$. 